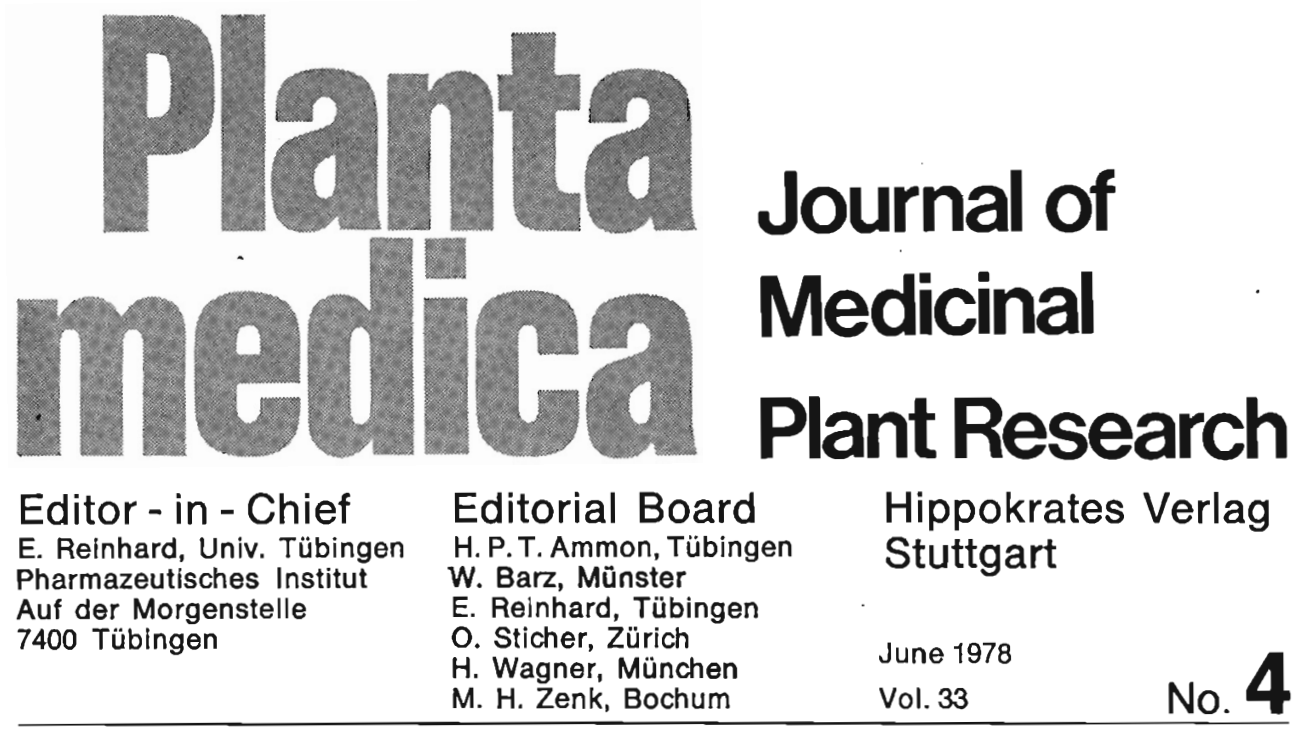

Review Arriche

\title{
Strukturaufklärung von Iridoidglykosiden
}

\section{Structure Elucidation of Iridoidglykosides \\ H. Rimpler}

Institut für Pharmazeutische Biologie, Universität Freiburg, Bundesrepublik Deutschland.

Key Word Index: Iridoids; Structure; Configuration; Conformation; NMR Spectrometry; Mass Spectrometry.

\section{Abstract}

The literature concerning the determination of structure, configuration and conformation of iridoids is reviewed. The use of NMR spectroscopy for the determination of configuration and preferred conformation of the cyclopentanering is discussed in some detail.

Unter Iridoiden versteht man Monoterpene mit einem Hexahydro-cyclopenta[c]pyran-Ringsystem und davon ableitbare Verbindungen. Die Stoff- 


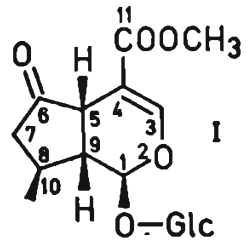

Verbenalin [1]<smiles>CC(Cl)(Cl)O[C@H]1OC=C[C@@H]2[C@H](O)C=C(CO)[C@@H]21</smiles>

Aucubin $[2,3,4]$<smiles>[CH]OCC1=CO[C@H](OC(=O)CC(C)C)[C@H]2C(CO)[C@@H](O)C[C@H]12</smiles>

Patrinosid [10]<smiles>CC(Cl)(Cl)O[C@@H]1[C@@H]2OC=C[C@@H]1[C@@H](O)[C@H]1O[C@@H]21</smiles>

Unedosid [6,7]<smiles>CC1=CO[C@H](OC(F)(Cl)Cl)[C@H]2[C@@H](O)C[C@@H](O)[C@]12O</smiles>

Lamiol [8]<smiles>OCC1=CO[C@H](OC(Cl)Cl)[C@@H]2C=C[C@@H](O)[C@H]12</smiles>

Decalosid [5]<smiles>CC1=C[C@H](O)[C@H]2OC=C[C@H](OC3CCCCC3)[C@H]12</smiles>

10-0-Glucosyl-Aucubin [11]

Ixorosid [9]

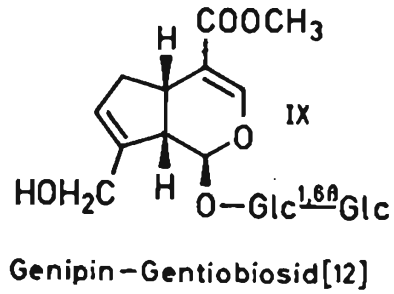

gruppe wird normalerweise in Iridoide im engeren Sinne und Seco-Iridoide unterteilt. Iridoide, die aus mehreren Monoterpeneinheiten aufgebaut sind, oder die außer dem Iridoidanteil noch andere Bausteine enthalten, werden hier als gesonderte Gruppe aufgeführt. Die folgenden Abbildungen zeigen einige Glykoside aus den genannten Stoffgruppen. Die Beispiele sind so ausgewählt, daß sie einen Eindruck von den möglichen Strukturvariationen geben: Bei den Iridoiden im engeren Sinne kann der
Grundkörper 10 (I), 9, (II ; III) oder 8 (IV) C-Atome enthalten. Das C-11 liegt normalerweise als Carboxymethyloder Carboxylgruppe vor, aber auch andere Oxydationsstufen kommen vor (III, V, VI). An funktionellen Gruppen sind vor allem Hydroxy -, Epoxy-(IV) und Carbonyl-(I) Gruppen sowie weitere $\mathrm{C}=\mathrm{C}$-Doppelbindungen (III) vorhanden. Als Zuckerkomponente tritt in allen bisher bekannten Iridoiden Glucose auf; Zahl und Stellung der Glucosereste ist jedoch variabel. Neben 1-Mono- 


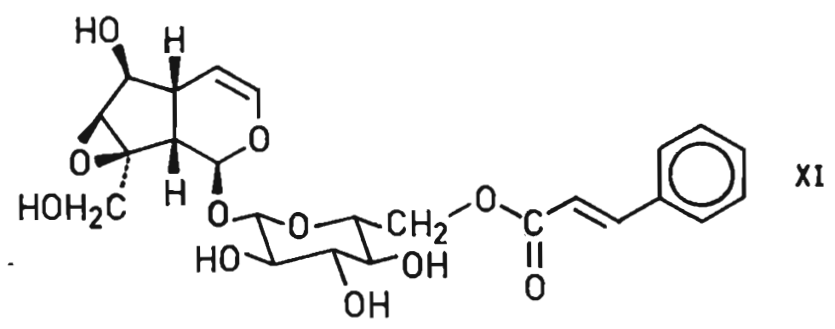

Picrosid I [15]

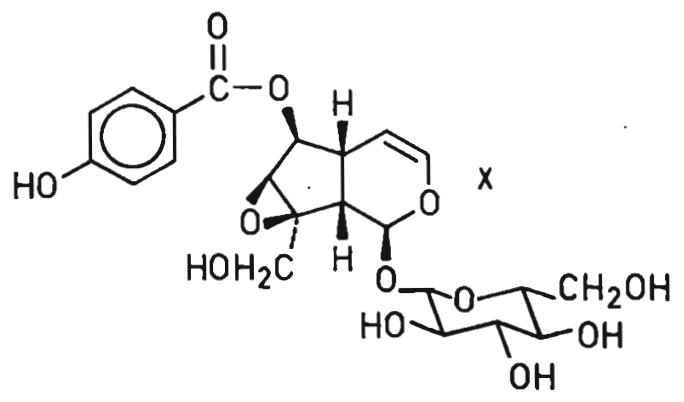

Catalposid $[13,14]$

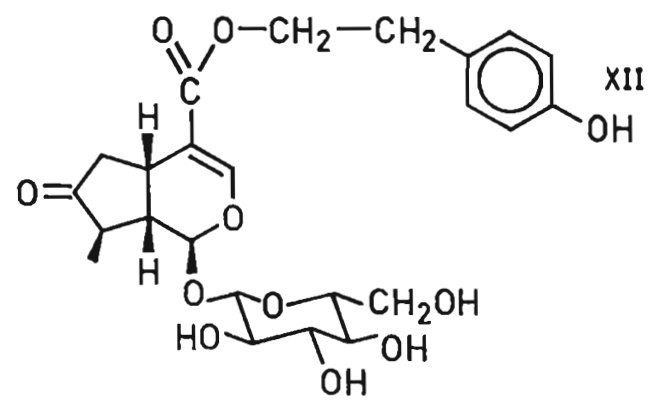

Syringopicrosid [16]

glucosiden kommen 11-Monoglucoside (VII), Diglucoside (VIII) und Bioside (IX) vor. Auch Ester von Iridoiden sind relativ häufig; dabei kann der Acylrest entweder an das Aglykon (X) oder an den Zuckerrest (XI) gebunden sein, oder eine Carboxylgruppe am C-4 ist mit einem Alkohol verestert (XII).
Bei den Seco-Iridoiden ist der Cyclopentanring aufgespalten, wobei eine Sauerstoff-Funktion am C-7 und eine Doppelbindung entweder in 8(9)-(XIII) oder in 8(10)-Stellung (XIV) entstanden ist. Häufig enthalten diese Verbindungen Lakton- (XV) oder Cyclohalbacetal-(XVI) Gruppen. Auch Seco- 
<smiles>C=CC1(CC=O)C(C(=O)OC)=COC(OCl)C1(C)CCO</smiles>

Lonicerosid [19]<smiles>C=CC1C2=CCOC(=O)C2=CO[C@H]1OC(C)=O</smiles>

Gentiopikrin [20]

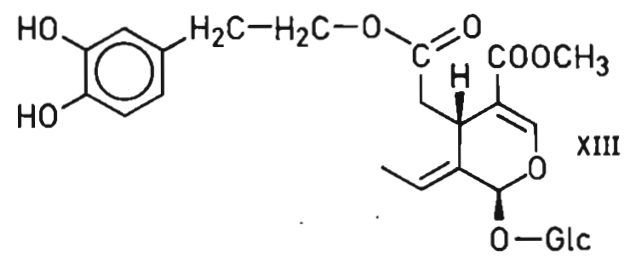

Oleuropein $[17,18]$<smiles>C=CC1(C)C(OC(C)=O)OCC2=COC(=O)CC23CC(OC(=O)C(C)=CCCC(C)=CCO)CC31</smiles>

Foliamenthin [23]

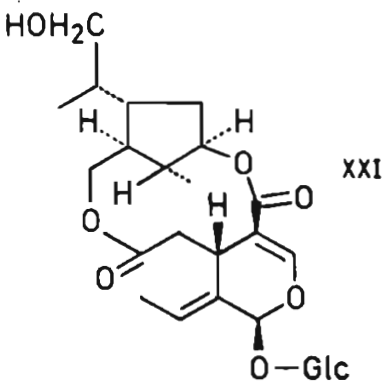

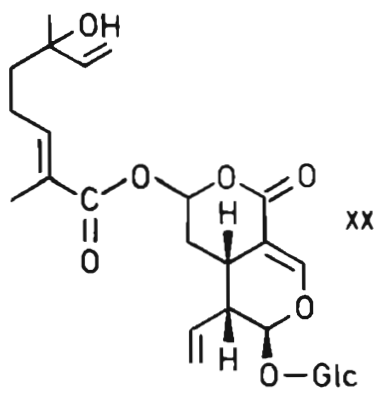

Menthiafolin [24]

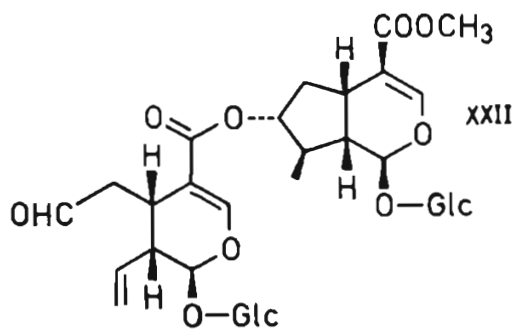

Cantleyosid [26] 


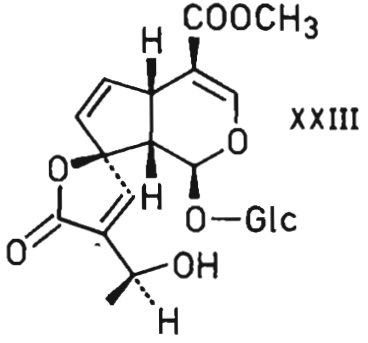

Plumierid [27]

Iridoide kommen häufig als Ester vor, wobei im wesentlichen die gleichen Variationen möglich sind wie bei den Iridoiden. Die letztgenannten Verbindungen leiten zu einer Gruppe der komplexen Iridoide über, bei denen ein Secoiridoidanteil mit einem mehr oder weniger iridoidartigen Monoterpen esterartig verknüpft ist (IXX, XX, XXI, XXII). Eine weitere Gruppe komplexer Iridoide enthält außer dem Monoterpenanteil noch einen über C-CBindungen verknüpften $\mathrm{C}_{4}$-Körper, der aus 2 Acetateinheiten entstanden ist (XXIII).

\section{Bestimmung der Struktur des Grundgerüstes}

Hinweise auf die Zugehörigkeit einer neuen Verbindung zu den Iridoiden erhält man am einfachsten durch Farbreaktionen und spektroskopische Methoden.

Als Farbreaktion eignet sich besonders gut die von Trim und Hrll [28] eingeführte „Pseudoindikan“-Reaktion. Das Reagens besteht aus $\mathrm{CuSO}_{4}$, Salzsäure und Eisessig und gibt mit vielen Iridoiden beim Erhitzen mehr oder weniger intensive, häufig blaue bis violette Färbungen [29]. Auch das Erhitzen von Iridoiden mit Mineralsäuren ist zum

Nachweis geeignet. Es scheider sich ein dunkelgefärbter Niederschlag $a b$, der durch Zersetzung des instabilen Aglykons entsteht [30]. Vor der Bildung des Niederschlages tritt häufig eine blaue Färbung der Lösung auf, die auf die Bildung von Salzen der entsprechenden Cyclopenta[c]pyran-Derivate zurückzuführen ist [31].

\section{Tabelle I}

Spektroskopische Nachweismöglichkeiten der Enoläther-Doppelbindung von Iridoiden [30]

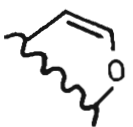

$\mathrm{UV}: \lambda \max \quad 188-210 \mathrm{~nm}$ IR: $v \max \quad 1640-1660 \mathrm{~cm}^{-1} \quad 1640-1660 \mathrm{~cm}^{-1}$ NMR: $\delta \quad 6,4-6,6 \mathrm{ppm} \quad 7,4-7,8 \mathrm{ppm}$

Spektroskopisch läßt sich vor allem die charakteristische Enoläther-Doppelbindung relativ eindeutig nachweisen (Tab. 1). Der Beweis für die Struktur und Konfiguration des Grundkörpers wird dann in der Regel durch Abbau zu Cyclopentancarbonsäuren oder durch Korrelation mit bereits bekannten Iridoiden geführt. Der oxydative Abbau zu Cyclopentancarbonsäuren, die durch Synthese zugänglich sind, wurde z. B. bei der Strukturaufklärung von Aucubin angewandt (Abb. 1) und führte zur Aufstellung der ersten korrekten Strukturformel für diese Verbindung [2, 32]. Inzwischen' sind eine Vielzahl von Iridoiden bekannt und es gelingt fast immer, ein neues Iridoid durch relativ wenige Reaktionen in eine bereits bekannte Verbindung zu überführen, 

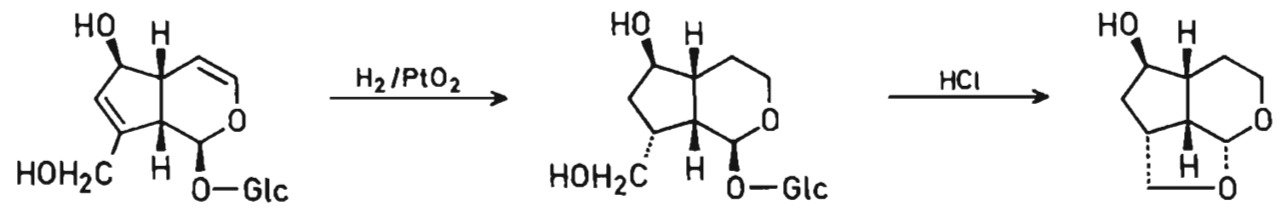

Aucubin

Abb. 1. Abbau von Aucubin $[2,32]$
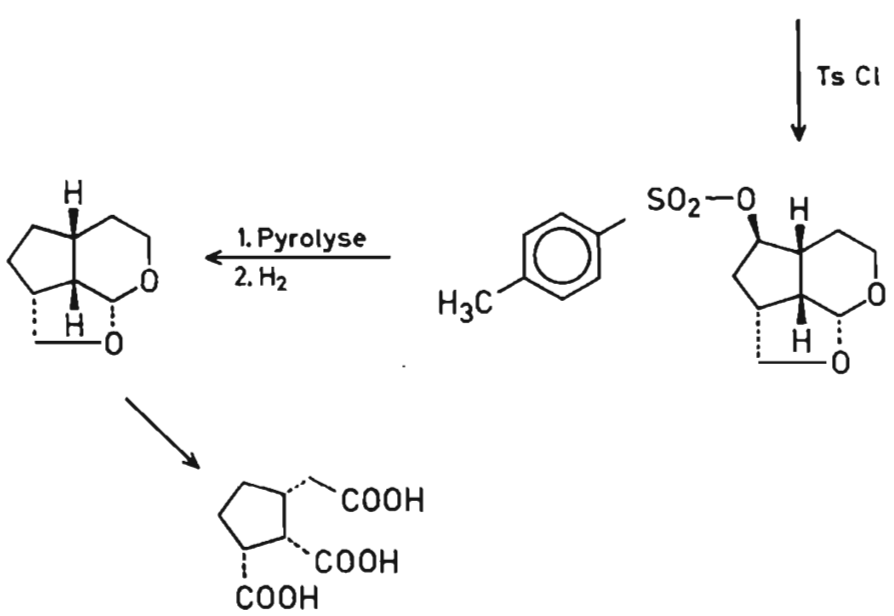

ohne den Zuckerrest abzuspalten oder den Dihydropyranring zu verändern. Dies hat den Vorteil, daß die Konfiguration auch am $\mathrm{C}-1$ und $\mathrm{C}-1^{\prime}$ erhalten bleibt, und daß sich die Umsetzungen in der Regel mit geringen Substanzmengen durchführen lassen. Im Folgenden sollen die häufiger verwendeten Reaktionstypen an einigen Beispielen erläutert werden.

Die Birch-Reduktion mit Lithium in flüssigem Ammoniak wird vor allem zur Entfernung allylischer Hydroxygruppen eingesetzt. Aus Aucubin entsteht bei vollständiger Reduktion Bisdesoxy-aucubin [3]. Führt man die Reduktion unter milden Bedingungen durch, so entsteht neben Bisdesoxy-aucubin auch 10-Desoxyaucubin, nicht aber das isomere 6-Desoxyaucubin [33] (Abb. 2). Das zeigt, daß primäre $\mathrm{Hy}-$ droxygruppen leichter als sekundäre angegriffen werden. Bei der BIRCH-Reduktion können auch eine Reihe weiterer Reaktionen ablaufen. So entstehen bei der Reduktion von Galiridosid drei Verbindungen, die als Reptosid, 5-Hydroxy-bisdesoxyaucubin (XXIV) und Bisdesoxy-aucubin identifiziert wurden (34, 35, 36] (Abb. 3). Der Epoxidring ist also aufgespalten worden und die Sauerstoff-Funktion am C-8 wurde z.T. eliminiert. Die BIRCH-Reduktion wurde z. B. bei der Strukturaufklärung von Aucubin [3], Melittosid [37], Galiridosid [34], Reptosid [36], Antirrhinosid [38], und 6-Desoxyharpagid eingesetzt. Dabei wurden entweder die unbekannten Iridoide in Bisdesoxyaucubin überführt, oder Zwischenstufen der Reduktion von bekannten Iridoiden erwiesen sich als identisch mit einer neuen Verbin- 
$A b b$. 2. Reduktion von Aucubin mir $\mathrm{Li} / \mathrm{NH}_{3}[3,33]$<smiles>OCC1=C[C@@H](O)[C@H]2C=CO[C@H](OCl)[C@H]12</smiles>

Aucubin

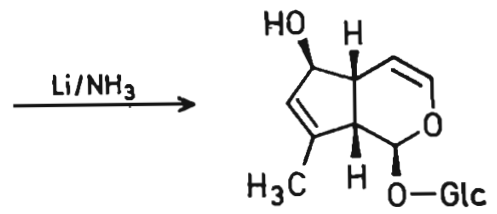

Linarid

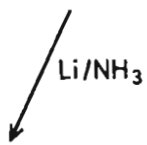<smiles>CC1=CC[C@H]2C=CO[C@H](OC(Cl)Cl)[C@H]12</smiles>

Bisdesoxy - Aucubin<smiles>C[C@]12O[C@H]1C[C@]1(O)C=CO[C@H](OC(Cl)(Cl)Cl)[C@H]12</smiles>

Galiridosid<smiles>C[13CH3]</smiles>

$\mathrm{Li} / \mathrm{NH}_{3}$<smiles>C[C@]1(O)CC[C@@]2(O)C=CO[C@@H](O[Ge])[C@H]12</smiles>

Reptosid

Abb. 3. Reduktion von Galiridosid mit $\mathrm{Li} / \mathrm{NH}_{3}[34,35,36]$

dung. Die tertiäre allylische $\mathrm{OH}-\mathrm{Grup}-$ lingt beim Hastatosid [39] durch eine pe am C-5 läßt sich allerdings nur sehr modifizierte Clemensen-Reduktion [40] schwer entfernen, wie die geringen Aus- in recht guter Ausbeute (Abb. 4). Diese beuten an Bisdesoxyaucubin zeigen. Die Methode scheint jedoch nicht bei allen Entfernung der 5-Hydroxygruppe ge- Iridoiden anwendbar zu sein. 
<smiles>COC(=O)OC1OC=C(OC(C)=O)[C@H]2C(C)CC(=O)[C@@]12O</smiles>

Hastatosid

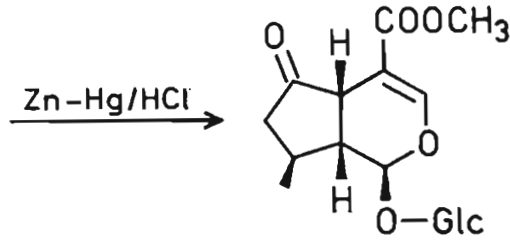

Verbenalin
Abb. 4. Reduktion von Hastatosid [39]

Bei der katalytischen Hydrierung kannt war, mit anderen Iridoiden (Lokönnen ebenfalls allylische Hydroxy- ganin [42], Monotropein [41], Aucubin oder Acyloxygruppen entfernt werden. [43]) und Secoiridoiden (Swerosid [44]) Z. B. entsteht bei der vorsichtigen Hyd- korrelierten. Auch bei dieser Reaktion

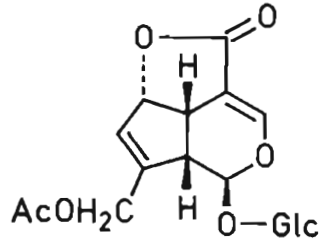

Asperulosid

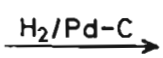<smiles>[CH]O[C@H]1OC=C(C(=O)O)[C@@H]2CCC=C(C)[C@H]12</smiles>

Abb. 5. Katalytische Hydrierung von Asperulosid [41]

rierung von Asperulosid die Verbindung werden primäre Acyloxygruppen offen$\mathrm{XXV}$ (Abb. 5) über die INOUYE und bar rascher abgespalten als sekundäre, seine Arbeitsgruppe das Asperulosid, wie die Hydrierung von XXVI zu dessen Struktur und Konfiguration be- XXVII zeigt (Abb.6), die bei der Struk-
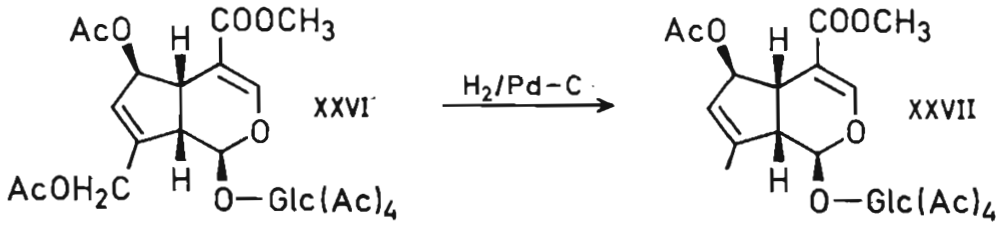

POCl 3 /Pyridin

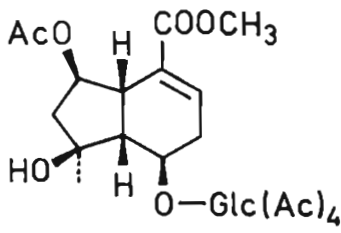

Abb. 6. Korrelation von Shanzhisidmethylester-pentaacetat [XXVII] mit Scandosidmethylesterhexaacetat [XXVI] 
<smiles>[CH]O[C@@H]1OC=C(C(C)=O)[C@H]2[C@H](C)[C@@H](O)C[C@@H]21</smiles>

Loganin

1. Emulsin

2. $\mathrm{MeOH}_{/} \mathrm{BF}_{3}$<smiles>CO[C@H]1OC=C(C(C)=O)[C@H]2C[C@H](O)[C@@H](C)[C@H]12</smiles>

$\downarrow \mathrm{MsCl}$ PY<smiles>CC(=O)C1=CO[C@@H](OC(Cl)(Cl)Cl)[C@H]2C(=O)C[C@H](C)[C@H]12</smiles>

Verbenalin

1. $\mathrm{NaBH}_{4}$

2. Emulsin

3. $\mathrm{MeOH} / \mathrm{BF}_{3}$

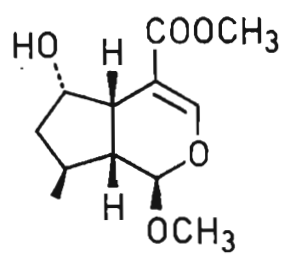

$\mathrm{MsCl} / \mathrm{PY}$<smiles>COC(=O)C1=CO[C@H](OC)[C@@H]2C(C(=O)OC)=CO[C@H](OC)[C@H]1C2COS(C)(=O)=O</smiles><smiles>COC(=O)C1=CO[C@H](OC)[C@H]2C(C)=CC[C@H]12</smiles><smiles>CC1CC1</smiles><smiles>Cc1cccc(C)n1</smiles><smiles>CO[C@H]1C=C(C(C)=O)[C@H]2C=C[C@@H](C)[C@@H]21</smiles>

$A b b .7$. Korrelation von Loganin mit Verbenalin [46] 


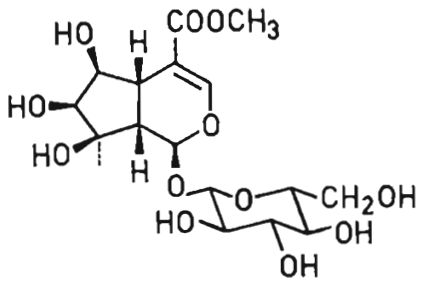

Lamalbid

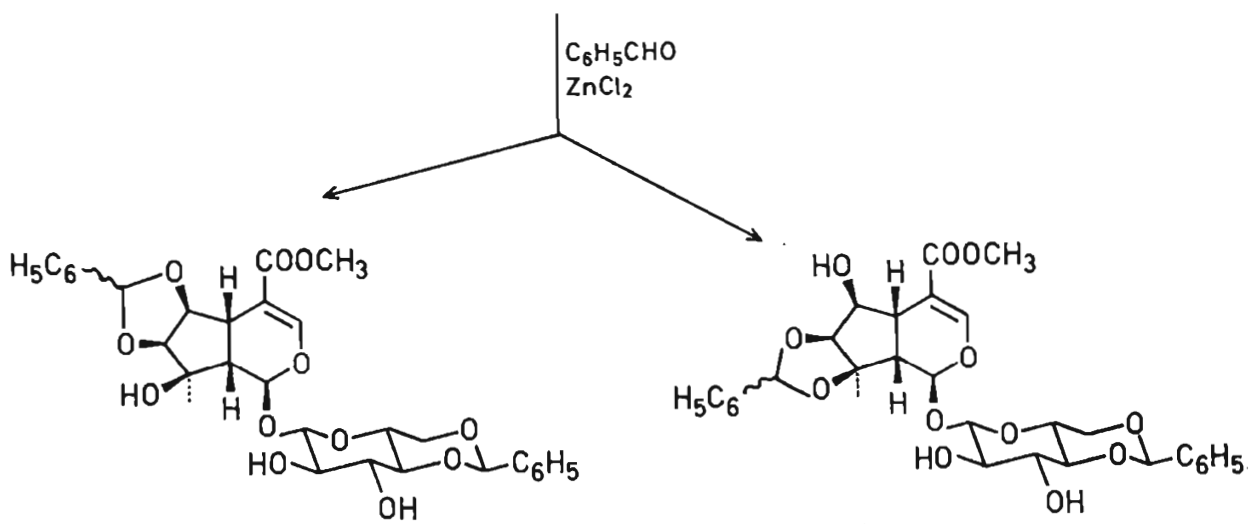

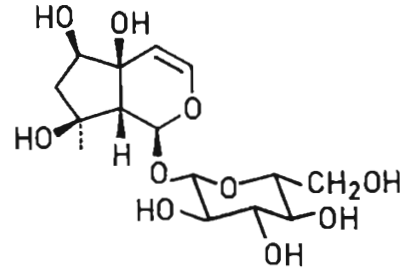

Harpagid

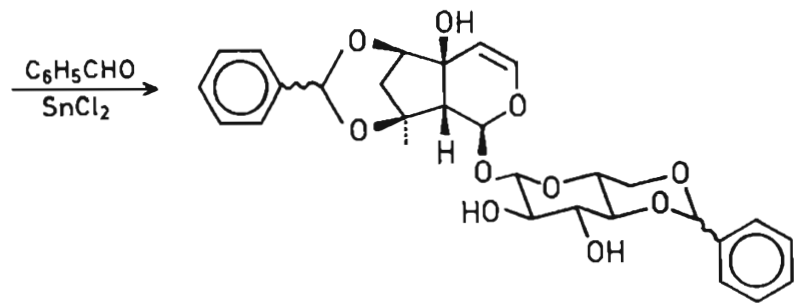

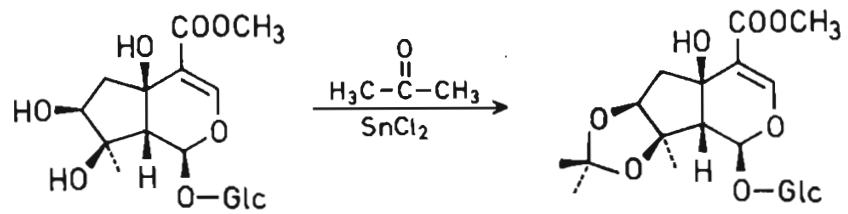

Lamiid

Abb. 8. Benzyliden- und Isopropylidenderivate von Iridoiden [47, 48, 49] 
turaufklärung von Shanzhisid durchgeführt wurde [45].

Nichtallylische Hydroxygruppen können durch Eliminierung entfernt werden. Entweder dehydratisiert man den Alkohol direkt, etwa durch Umsetzung mit $\mathrm{POCl}_{3}$ (Abb. 6) oder man verestert zunächst und spaltet dann mit Basen die Säure ab. Als Beispiel für eine derartige Reaktionssequenz ist die Korrelation von Loganin und Verbenalin [46] (Abb. 7) angegeben.

Korrelationsprobleme können häufig auch durch Einführung weiterer Sauerstoff-Funktionen gelöst werden. Beispiele für derartige Reaktionsfolgen findet man vor allem in den Arbeiten des Arbeitskreises von INOUYE [41-44].

\section{Bestimmung funktioneller Gruppen}

Art, Zahl und Stellung von funktionellen Gruppen läßt sich meist spektroskopisch und durch Darstellung von Derivaten ermitteln. So lassen sich z. B. Carbonylgruppen und Epoxide im IRSpektrum nachweisen. Epoxide geben darüber hinaus eine positive Reaktion mit Natriumthiosulfat/Phenolphtalein [13]. Art und Zahl der Hydroxygruppen weist man am besten durch Acetylierung und anschließende NMR- und Massenspektrometrie nach. Die Bildung von Acetoniden und Benzylidenderivaten beweist die cis-Stellung zweier $\mathrm{Hy}$ droxygruppen am Cyclopentanring. Dabei können Acetonide nur aus 1,2Diolen, Benzylidenderivate dagegen aus 1,2 und aus 1,3-Diolen gebildet werden (Abb. 8). Alle weiteren Informationen über die Stellung funktioneller Gruppen erhält man normalerweise durch Analyse der Massen- und NMRSpektren des Iridoids und seiner Deri- vate. Die wichtigsten Fragmentierungen von Iridoiden im Massenspektrometer [50] sind in Abb. 9 am Beispiel des Verbenalins dargestellt. Das Molekülion ist zwar meist nicht nachweisbar, doch läßt sich die Molmasse des Aglykons aus den Massenzahlen der Ionen A und $C$ ableiten. Aus der Massenzahl des Ions $B$ ergibt sich die Art der Substituenten am C-4 und C-5. Aus $\mathrm{C}_{9}$-Iridoiden ohne Substituenten am C-4 und C-5, z. B. Aucubin [50], entsteht ein Ion B mit der Massenzahl 81. Eine 5-Hydroxygruppe, z. B. in Stilbericosid [7], erhöht die Massenzahl um 16 Einheiten. Aus den Massenspektren läßt sich auch ableiten, ob bestimmte funktionelle Gruppen, z. B. Isopropyliden- oder Acylreste mit dem Zucker oder mit dem Aglykon verknüpft sind: $z$. B. bildet sich beim Zerfall von Picrosid I-Acetat ein Ion C mit der Massenzahl 267 und ein Ion D mit der Massenzahl 419. Daraus geht eindeutig hervor, daß der Cinnamoylrest an den Zuckeranteil gebunden ist (Abb. 10). Aus der weiteren Fragmentierung von Ion $D$ kann man schließen, daß die Bindungsstelle wahrscheinlich das C-6 ist. Das Massenspektrum von Picrosid-II-Acetat (Abb. 11) zeigt demgegenüber ein Ion $C$ mit der Massenzahl 417 und ein Ion D mit der Massenzahl 331; der Cinnamoylrest muß daher mit dem Aglykon verknüpft sein [51].

Wesentlich mehr Informationen erhält man aus den NMR-Spektren. Das Spektrum eines Iridoids kann man grob in drei Abschnitte unterteilen [30]:

1. den Bereich der Protonen an gesättigten Kohlenstoffatomen, welche nicht mit Heteroatomen verknüpft sind, etwa 0,9-3,5 ppm. 

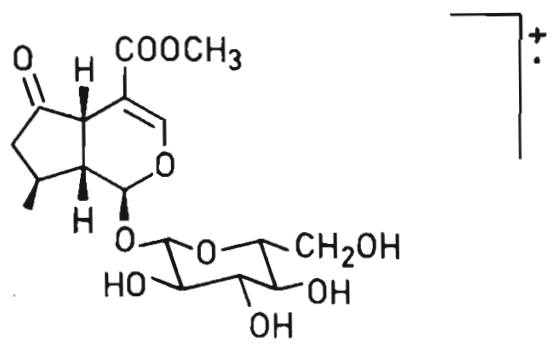

$\mathrm{m} / \mathrm{e} 388$
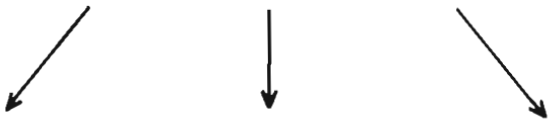

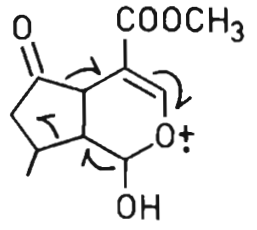

A

$\mathrm{m} / \mathrm{e} 226$<smiles>CC1CCC(=O)[C@H](O)C1C</smiles><smiles>CC(=O)C1=CC=C[C+]=C1</smiles>

B<smiles>CC(=O)C1=C[O+]=CC2C(C)CC(=O)C12</smiles>

C

$\mathrm{m} / \mathrm{e} 209$<smiles>OCC1C=[O+]C(CO)C(O)C1O</smiles>

D

$\mathrm{m} / \mathrm{e} 163$

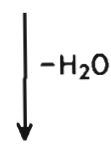

$\mathrm{m} / \mathrm{e} 145$

$\mathrm{m} / \mathrm{e} 139$

Abb. 9. Massenspektren von Verbenalin: Wichtige Fragmentierungswege [50]

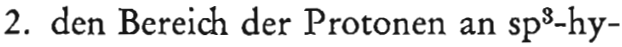
bridisierten Kohlenstoffatomen, welche mit Sauerstoff verbunden sind: etwa 3,9-5,8 ppm.

3. den Bereich der Protonen, die an $\mathrm{sp}^{2}$-hybridisierten Kohlenstoffatomen stehen: etwa 4,9-7,8 ppm.
Die Signale im 3. Bereich sind meist ohne Schwierigkeiten interpretierbar: Bei tiefstem Feld erscheint das Signal für das $\mathrm{C}(3)-\mathrm{H}$ (Tab. 1). Es tritt je nach Art der Substituenten am C-4 und C-5 als Singlett (C-4 und C-5 substituiert), Dublett (C-4 oder C-5 substituiert) 


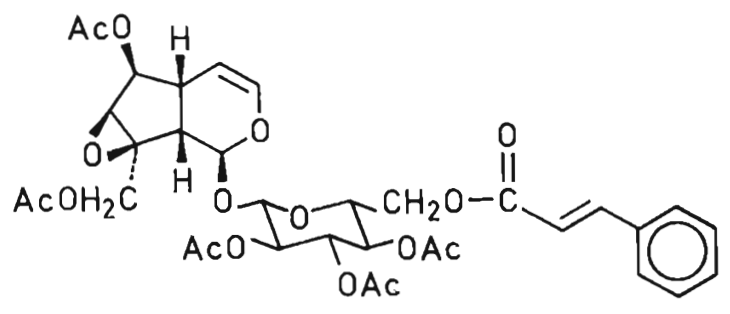<smiles>CC(=O)OC1C=[O+]C(C)C(=O)C1</smiles><smiles></smiles>

$\mathrm{m} / \mathrm{e} 317$ $\mathrm{m} / \mathrm{e} 359$<smiles>CC1C=[O+]C(COC(=O)C=Cc2ccccc2)C(=O)CC1</smiles>

$\mathrm{m} / \mathrm{e} 257$

Abb. 10. Massenspektrum von Picrosid I-Acetat: Wichtige Fragmentierungen [51] 

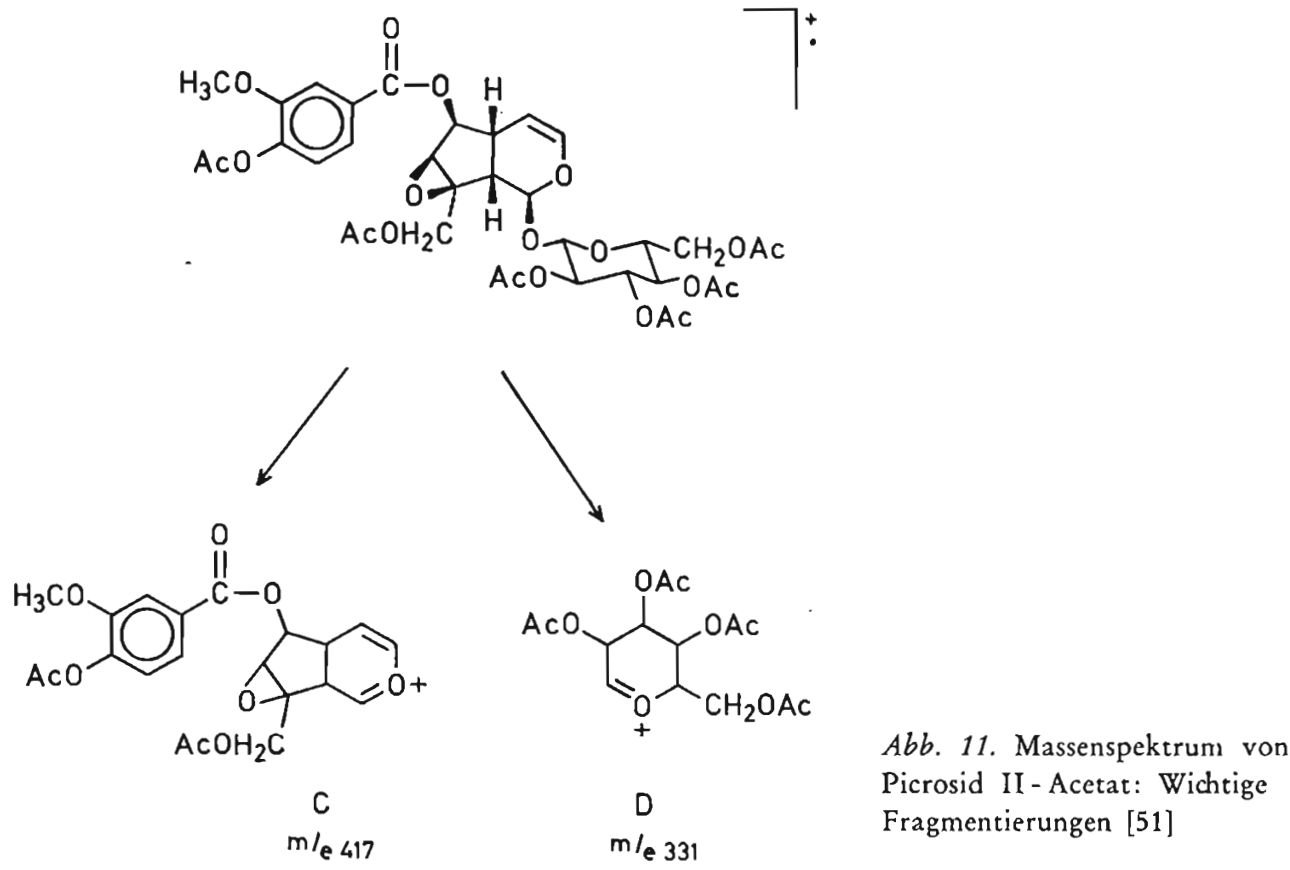

oder Doppeldublett (C-4 und C-5 unsubstituiert) auf. Sind weitere Wasserstoffatome an dem C-4-Substituenten vorhanden, so ist die Linienzahl des $\mathrm{C}(3) \mathrm{H}$-Signals entsprechend höher. Ist ein $\mathrm{C}(4) \mathrm{H}$ vorhanden, so findet man das entsprechende Signal bei höherem Feld, wo es z. T. schon von den Signalen des 2. Bereichs überlappt wird. Zusätzliche Doppelbindungen lassen sich durch weitere Signale in diesem Bereich erkennen.

Auch der 1. Bereich ist meist recht gut interpretierbar und liefert wichtige Strukturinformationen. Bei höchstem Feld erscheint das Signal für die Methylgruppe am C-8, aus dessen chemischer Verschiebung und Multiplizität man auf die Art des zweiten Sustituenten am C-8 (z. B. $\mathrm{H}, \mathrm{OH}$, oder O-Acetyl) schließen kann. Die Brückenkopf-H-Atome am C-9 und C-5 sind am stärksten entschirmt; ihre Signale liegen bei tieferem
Feld. Wenn beide H-Atome im Molekül vorhanden sind, so sind ihre Signale häufig komplizierte Multipletts die sich bei $60 \mathrm{MHz}$-Spektren nur durch Doppelresonanzexperimente analysieren lassen. Durch vollständige Analyse dieser Signale erhält man Informationen über die Substituenten am C-8, C-1 und C-6, die nicht nur für die Strukturaufklärung, sondern auch für die Bestimmung der relativen Konfiguration (s. u.) wichtig sind. Die übrigen Signale in diesem Bereich (z. B. von $\mathrm{C}(8)-\mathrm{H}$ oder $\mathrm{CH}_{2^{-}}$ Gruppen im Cyclopentanring) lassen sich meist durch Doppelresonanz zuordnen und mehr oder weniger vollständig analysieren.

Die Interpretation des mittleren Bereichs wird durch die Signale der Zukkerprotonen und des Wassers erschwert. Fast immer zu erkennen ist das Signal des $\mathrm{C}(1)-\mathrm{H}$, dessen Kopplungskonstante 
für die Konfigurationsbestimmung wichtig ist, bei etwa 4,8-5,8 ppm. Häufig ist auch das bei etwas höherem Feld liegende C-1'-Signal, aus dessen Kopplungskonstante man die Konfiguration am anomeren C-Atom des Zuckers bestimmen kann, nachweisbar. Signale von Protonen, die neben Hydroxy- oder Epoxidgruppen stehen, werden häufig von Zuckerprotonen-Signalen überdeckt. In diesen Fällen hilft man sich mit der Herstellung geeigneter Derivate, die dann NMR-spektroskopisch untersucht werden. Durch Acetylierung werden z. B. die Signale aller Protonen neben sekundären $\mathrm{OH}-\mathrm{Gruppen}$ um etwa $1 \mathrm{ppm}$ nach tieferem Feld verschoben und bilden dann eine häufig nicht interpretierbare Signalgruppe bei etwa 5 ppm. Bei primären Hydroxygruppen werden die Signale der benachbarten
Protonen aber nur um etwa 0,5 ppm verschoben; sie sind daher meist gut von den übrigen Signalen abgesetzt und können interpretiert werden. Die Signale von Protonen an Oxiranringen werden praktisch nicht verschoben und sind dann ebenfalls gut erkennbar. Für Iridoide, die mehrere Hydroxygruppen im Aglykon enthalten, sind Acetonid-Acetate oder Benzylidenverbindungen besonders gut geeignet, da hier nur ein Teil der Signale verschoben wird und dadurch meist ein sehr übersichtliches Spektrum resultiert. (Abb. 12). Die Analyse eines solchen Spektrums liefert also im Zusammenhang mit den bereits auf anderem Wege erhaltenen Kenntnissen über Summenformel und funktionelle Gruppen folgende Daten:

1. Art und Zahl funktioneller Gruppen.
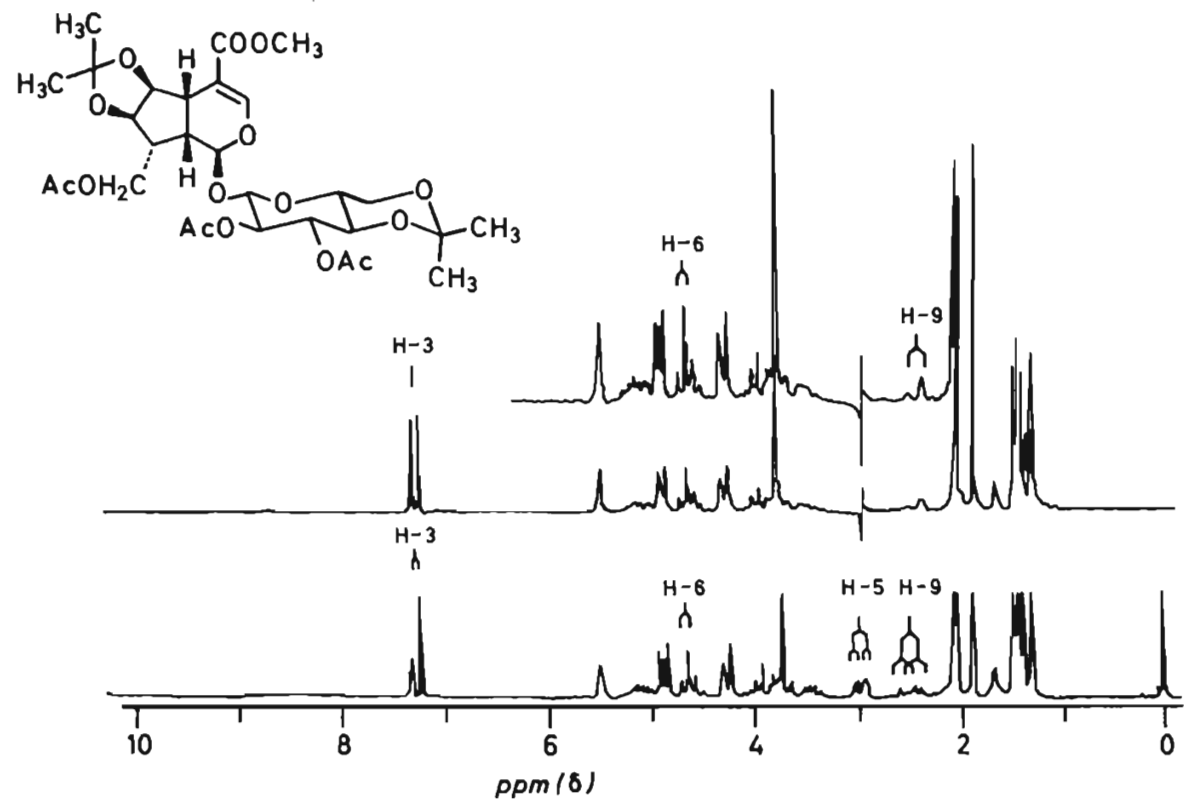

Abb. 12. NMR-Spektrum des Nyctanthosiddiacetonidtriacetates (Lösungsmittel $\mathrm{CDCl}_{3}$ ) vor (unten) und nach (oben) Entkopplung des $\mathrm{C}(5)-\mathrm{H}$ (bei 2,85 ppm) 
2. Stellung der funktionellen Gruppen am Grundgerüst.

Derartige Informationen lassen sich nicht nur aus ${ }^{1} \mathrm{H}-\mathrm{NMR}-$ Spektren sondern auch aus ${ }^{13} \mathrm{C}-\mathrm{NMR}$-Spektren [5256] erhalten. Darüberhinaus können NMR-Spektren auch zur Bestimmung der relativen Konfiguration herangezogen werden.

\section{Bestimmung der Konfiguration}

Wichtigste Voraussetzung für die Bestimmung relativer Konfigurationen aus NMR-Daten ist, daß die untersuchte Verbindung - zumindest überwiegend in einer bestimmten Konformation vorliegt. Diese Voraussetzung ist bei vielen Cyclopentanderivaten nicht erfüllt: Unsubstituiertes Cyclopentan besteht aus einem Gemisch von Konformeren die sich laufend ineinander umwandeln. Diese Umwandlungen lassen sich am übersichtlichsten als Pseudorotationszyklus (cycle of pseudorotation) darstellen, bei dem 10 Briefumschlag- (envelope) und 10 Halbsessel (twist) Konformationen (Abb. 13) in bestimmter Reihenfolge durchlaufen werden. Während im unsubstituierten Cyclopentan alle Konformeren energetisch gleichwer-
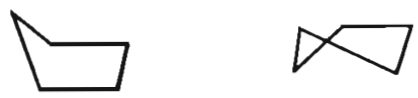

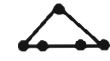

$\checkmark$

ENVELOPE

(Briefumschlag)

Abb. 13. Symmetrische Konformationen des Cyclopentans tig sind, werden bei Substitutionsprodukten, z. B. im Methylcycolpentan [57], oder in Methylcyclopentanolen [58] bestimmte Konformationen bevorzugt eingenommen, so daß die Pseudorotation auf eine - allerdings immer noch relativ große - Zahl von Konformationen eingeschränkt wird. Mit zunehmender Zahl von Substituenten nimmt dann die Zahl der bevorzugten Konformationen weiter ab. Z. B. liegt Lamalbid, bei dem alle Positionen des Cyclopentanrings mit mindestens einem Substituenten besetzt sind, bereits vorwiegend in einer Konformation vor [59]. Noch ausgeprägter ist dieses Verhalten bei Verbindungen, bei denen der Cyclopentanring mit anderen kleinen Ringen annelliert ist. So sind z. B. Cyclopentan-Epoxide so starr, daß sie weitgehend unabhängig von weiteren Substituenten immer in der gleichen Konformation vorliegen [60]. Acetonide und Benzylidenderivate des Cyclopentans sind etwas flexibler; ihre Konformation wird durch andere Substituenten beeinflußt. Häufig ist aber auch bei ihnen eine Konformation deutlich bevorzugt [60]. Acetonid-Acetate und die entsprechenden Benzylidenderivate von Iridoiden eignen sich daher gut für die NMR-spektroskopische Konfigurationsbestimmung. Zusammenfassend läßt sich also sagen: Die Konfigurationsbestimmung durch NMR verspricht Erfolg, wenn die Iridoide eine Epoxidgruppe enthalten, als Acetonide oder Benzylidenderivate vorliegen oder möglichst viele Substituenten am Cyclopentanring vorhanden sind.

Zur Bestimmung der Konfiguration können im Prinzip zwei verschiedene Kriterien herangezogen werden, die 
chemischen Verschiebungen und die Verschiebungen von $8 \alpha$ - und $8 \beta$-AceKopplungskonstanten. Der Vergleich toxyderivaten miteinander verglichen chemischer Verschiebungen ist z. B. zur [45] (Abb. 14), oder die Ainderung der Bestimmung der Konfiguration von chemischen Verschiebung durch Acety8-Hydroxy-8-Methylsubstituierten Iri- lierung der $\mathrm{C}(8)-\mathrm{OH}$ Gruppe dient als doiden am C=8 herangezogen worden. Kriterium für die Zuordnung [61] (Abb. Dabei werden entweder die chemischen 15). Beide Methoden beruhen auf der
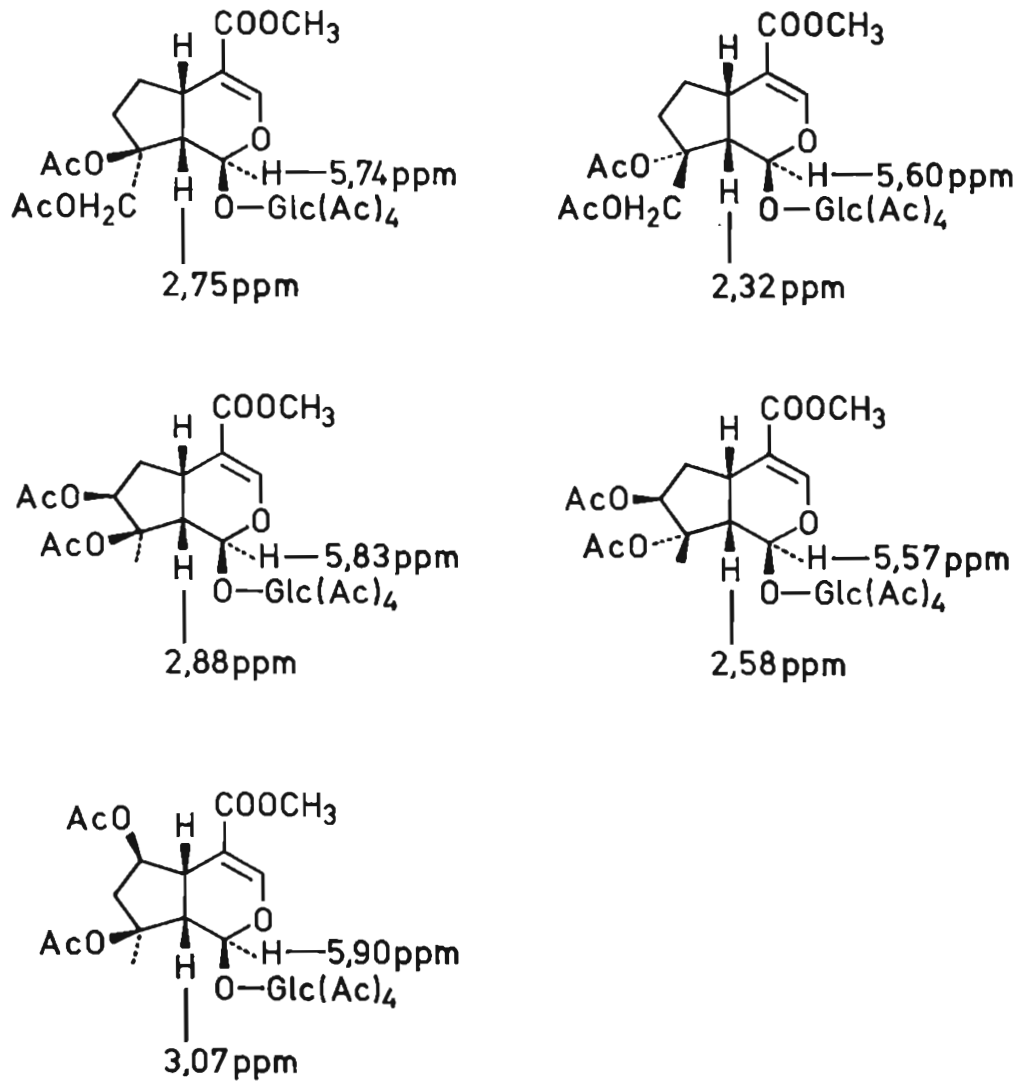

$A b 6$. 14. Vergleich chemischer Verschiebungen ( $\delta$-Werte) von $8 \alpha$ - und $8 \beta$-Acetoxy-Iridoiden [45]
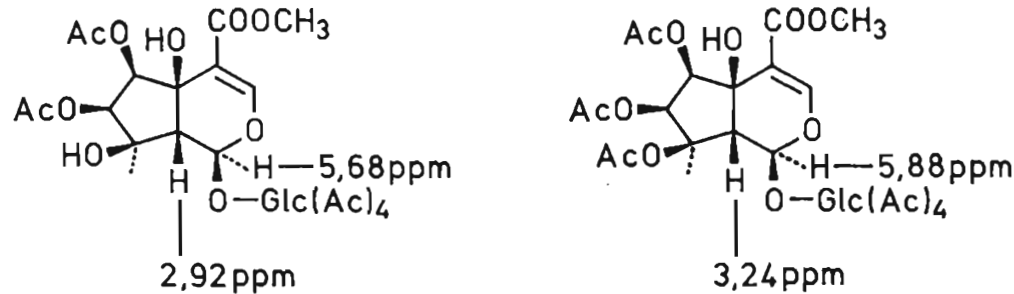

$A b b$. 15. Acetylierungsverschiebungen bei $8 \beta$-Hydroxy-Iridoiden [61] 
Beobachtung, daß eine $\beta$-ständige Acetoxygruppe am $\mathrm{C}-8$ die benachbarten Protonen am C-9 und C-1 deutlich entschirmt, während eine $8 \alpha$-Acetoxygruppe diesen Effekt nicht zeigt. $\mathrm{Die} \mathrm{C}(9)-\mathrm{H}-$ und $\mathrm{C}(1)=\mathrm{H}$-Signale von $8 \beta$-Acetoxyderivaten erscheinen daher bei tieferem Feld als die entsprechenden Signale von $8 a$-Acetoxyderivaten, und die Acetylierungsverschiebung beträgt bei $8 \beta$-Hydroxyverbindungen etwa je $0,2-0,4$ ppm für das $\mathrm{C}(9)-\mathrm{H}$ - und das $\mathrm{C}(1)-\mathrm{H}$ Signal. An Dreiding-Modellen kann man erkennen, daß eine $8 a$-Acetoxygruppe zwar mit dem $\mathrm{C}(1)-\mathrm{H}$, kaum aber mit dem $\mathrm{C}(9)-\mathrm{H}$ in Wechselwirkung treten kann. Aufgrund ähnlicher Uberlegungen kann man auch die Konfiguration von 7,8-Epoxiden bestimmen [42].

Die Konfigurationsbestimmung über Kopplungskonstanten beruht auf der Karplus-Gleichung, welche die Abhängigkeit der vicinalen Kopplungskonstante $\mathrm{J}_{\text {vic }}$ von dem Diederwinkel $\varphi$ нн beschreibt. Wir verwenden sie in der von Steyn und Sable [60] für Acetonide und andere polare Cyclopentanderivate benutzten Form

$$
\mathrm{J}_{\text {vic }}=\mathrm{A} \cos ^{2} \varphi \mathrm{HH}-\mathrm{B} \cos \varphi \mathrm{HH}+\mathrm{C}
$$

Ein gewisses Problem ist die Wahl der Werte für die „Konstanten“ A, B und C; denn die Kopplungskonstante hängt ja nicht nur vom Diederwinkel, sondern z. B. auch von der Elektronegativität der Substituenten ab. Man müßte daher streng genommen für jede Substanzgruppe einen speziellen Satz von Konstanten verwenden. $\mathrm{Da}$ die Gleichung aber sowieso nur relativ grobe Nährungswerte liefert, genügt es meist, die für mehr oder weniger ähnliche Verbin- dungen errechneten Werte zu verwenden. Wir benutzen daher die von Altona für Dihalogenderivate berechneten Werte, die auch von STEYN und SABLE verwendet wurden: $\mathrm{A}=9,5, \mathrm{~B}=$ $0,5, \mathrm{C}=0,4$.

Bei der Auswertung der Spektren gehen wir folgendermaßen vor:

1. Alle relevanten Kopplungskonstanten werden berechnet und als Funktion der Konformation graphisch dargestellt. Die für die Berechnung benötigten Diederwinkel haben wir der Arbeit von STEYN und SABLE entnommen. Aber auch die am Dreiding-Modell gemessenen Winkel sind verwendbar. Sie stimmen mit den berechneten Werten auf etwa $\pm 3^{\circ}$ überein.

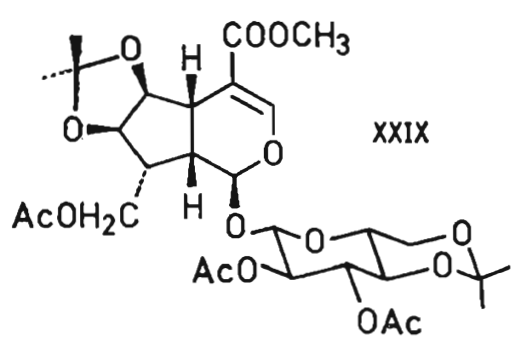

2. Die aus den Spektren entnommenen Kopplungskonstanten werden mit den theoretischen Werten verglichen, wobei zuerst die Kopplungskonstanten gewählt werden, welche besonders klein oder besonders groß sind. Beim Nyctanthosid-Acetonid-Acetat-(XXIX) gingen wir z. B. in der Reihenfolge $\mathrm{J}_{50}(<1$ $\mathrm{Hz}), \mathrm{J}_{89}(12 \mathrm{~Hz}), \mathrm{J}_{67}(5 \mathrm{~Hz})$ vor (Tab. 2). Hat man auf diese Weise die Zahl der möglichen Konformeren auf eine oder einige ähnliche eingeschränkt, so werden alle für diese Konformeren berechneten Kopplungskonstanten mit den gefundenen Werten verglichen. Auf 
Tabelle II

Ableitung der bevorzugten Konformation und der Konfiguration von Nyctanthosid-diacetonidtriacetat (XXIX) aus den Kopplungskonstanten [62].

\begin{tabular}{|c|c|c|}
\hline $\begin{array}{l}\text { gefundene Kopp- } \\
\text { lungskonstante }[\mathrm{Hz}]\end{array}$ & $\begin{array}{l}\text { Konformation (berechnete } \\
\text { Kopplungskonstante }[\mathrm{Hz}] \text { ) }\end{array}$ & Bemerkungen \\
\hline $\mathrm{J}_{5 \mathrm{~B}}<1$ & ${ }^{9} \mathrm{~V}^{9} \mathrm{~T}_{5} \mathrm{~V}_{5}{ }^{6} \mathrm{~T}_{5}{ }^{6} \mathrm{~V}^{6} \mathrm{~T}_{7} \mathrm{~V}_{7}(0,4-0,6)$ & $5 \beta 6 \alpha$ \\
\hline $\mathrm{J}_{8 \mathrm{~g}}=12$ & $\begin{array}{l}{ }^{6} \mathrm{~T}_{5}(8,9){ }^{6} \mathrm{~V}(9,4){ }^{6} \mathrm{~T}_{7}(8,9) \\
{ }^{9} \mathrm{~V}(9,4){ }^{9} \mathrm{~T}_{5}(8,5)\end{array}$ & $\begin{array}{l}8 \alpha 9 \beta \\
8 \beta 9 \beta\end{array}$ \\
\hline $\mathrm{J}_{\theta \prime \prime 7 a}=5$ & ${ }^{6} \mathrm{~V}(5,4)$ & ${ }^{9} \mathrm{~V}(9,4)$ \\
\hline
\end{tabular}

\section{Tabelle III}

Vergleich der für Nyctanthosid-diacetonid-triacetat (XXIX) gefundenen Kopplungskonstanten mit den nach STEYN und SABle [60] berechneten Werten.

\begin{tabular}{lcccccc}
\hline & $\mathrm{J} 5 \beta 6 \alpha$ & $\mathrm{J} 6 \alpha 7 \alpha$ & $\mathrm{J} 7 \alpha 8 \beta$ & $\mathrm{J} 8 \beta 9 \beta$ & $\mathrm{J} 9 \beta 5 \beta$ & $\mathrm{J} 9 \beta 1 \alpha$ \\
\hline $\begin{array}{l}\text { berechnet für } \\
{ }^{8} \mathrm{~V}\end{array}$ & $0,7 \mathrm{~Hz}$ & $5,4 \mathrm{~Hz}$ & $7,2 \mathrm{~Hz}$ & $9,4 \mathrm{~Hz}$ & $7,8 \mathrm{~Hz}$ & $2,5 \mathrm{~Hz}$ \\
gefunden & $<1 \mathrm{~Hz}$ & $5,0 \mathrm{~Hz}$ & $6,0 \mathrm{~Hz}$ & $12,0 \mathrm{~Hz}$ & $7,3 \mathrm{~Hz}$ & $2,0 \mathrm{~Hz}$ \\
\hline
\end{tabular}

diese Weise kommen wir z. B. bei XXIX zu folgendem Ergebnis (Tab. 3): Die Verbindung sollte bevorzugt in der ${ }^{6} \mathrm{~V}$ Konformation vorliegen, wobei die Wasserstoffatome am C-5 und C-6 sowie am C-7 und C-8 trans, die Wasserstoffatome am C-6 und C-7, am C-8 und C-9 sowie am C-9 und C-5 dagegen cis angeordnet sind. Damit ist die relative Konformation des Cyclopentanringes in XXIX bewiesen. Für jede Konformation des Cyclopentanringes sind aber nur wenige Konformationen des Dihydropyranringes möglich, die sich unter anderem in der Größe des Diederwinkels $\varphi_{19}$ voneinander unterscheiden. Man kann daher durch Vergleich der für diese Konformationen berechneten Kopplungskonstanten $\mathrm{J}_{19}$ mit den gefundenen Werten auf die Konformation des Dihydropyranringes und auf die
Konfiguration am C-1 schließen. Z.B. läßt sich der für XXIX gefundene Wert von $\mathrm{J}_{19}=2 \mathrm{~Hz}$ nur mit einer verdrillten Halbsessel-Konformation $\left(\mathrm{HCH}_{9}{ }^{1}\right)$ mit $\beta$-axial angeordnetem Zuckerrest und einer trans-Anordnung der $\mathrm{H}$-Atome an C-1 und C-9 vereinbaren. Damit ist die relative Konfiguration von XXIX und also auch von Nyctanthosid. bewiesen [62]. Auf ähnliche Weise sind die bevorzugten Konformationen von Lamalbid und Lamalbid-Derivaten bestimmt worden [59].

Die Kopplungskonstanten der Oxiran-Protonen in Cyclopentan-Epoxiden lassen sich nach diesem Verfahren nicht berechnen. Sie werden aber, bedingt durch das starre Ringsystem, durch andere Substituenten nur in Ausnahmefällen beeinflußt. Steyn und SAble [60] fanden für die cis-vicinale Kopplung 
der Oxiran-Protonen $\mathrm{J}=1,4 \mathrm{~Hz}$ und für die trans-vicinale Kopplung $\mathrm{J}=0,6$ $\mathrm{Hz}$. Für die Oxiran-Protonen im Mentzelosid XXX [63] und im Stilbericosid XXXI [7] findet man Kopplungskonstanten von $1,5 \mathrm{~Hz}$ und $\approx 0 \mathrm{~Hz}$ bzw. $1,5 \mathrm{~Hz}$ und $0,6 \mathrm{~Hz}$, die mit den angegebenen Werten sehr gut übereinstimmen und eine eindeutige Konfigurationszuordnung ermöglichen.

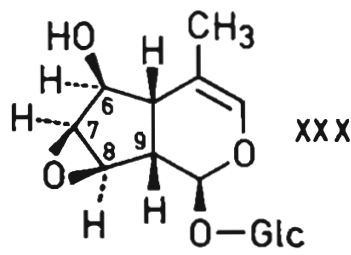

Mentzelosid [63]

Wenn die relative Konfiguration nach einem der genannten Verfahren bestimmt wurde, bleibt als letztes Problem noch die Bestimmung der absoluten Konfiguration. Die wichtigsten Methoden dafür sind die Röntgenstrukturanalyse, die partielle Racematspaltung nach Horeau, die Methode der Molrotationsunterschiede sowie die bereits erwähnte Korrelation neuer Verbindungen mit Iridoiden bekannter Konfiguration. Die Röntgenstrukturanalyse ist ein relativ aufwendiges Verfahren; es wird daher meist nur dann eingesetzt, wenn andere Methoden widersprüchliche Ergebnisse liefern. Mit dieser Methode ist die Konfiguration von Monotropein [64] und Loganin [65] bestimmt worden. Mit der Horeau-Methode [66] läßt sich die absolute Konfiguration sekundärer zyklischen Alkohole bestimmen. Man verestert die zu prüfende Verbindung mit racemischem $\alpha$-Phenylbutter- säureanhydrid. Dabei reagieren $\mathrm{R}-\mathrm{Al}$ kohole bevorzugt mit der $\mathrm{R}(-)$-Form und S-Alkohole bevorzugt mit der $\mathrm{S}(+)$-Form des Anhydrids. Das nicht verbrauchte Anhydrid wird anschlieBend hydrolysiert, die entstandene $a$ Phenylbuttersäure wird abgetrennt, und ihre optische Drehung wird bestimmt. Aus dem Vorzeichen der Drehung läßt sich dann auf die Konfigura-

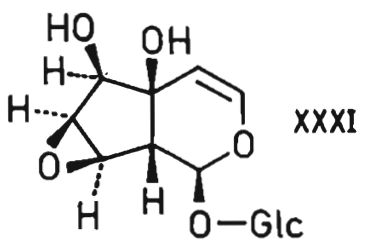

Stilbericosid [7]

tion des Alkohols schließen: isoliert man (+) $\alpha$-Phenylbuttersäure im Überschuß, wurde also das (-)-Anhydrid (= RForm) bevorzugt verbraucht, so hat der Alkohol R-Konfiguration. Ist die isolierte $\alpha$-Phenylbuttersäure linksdrehend, so hat der Alkohol S-Konfiguration. Mechanistische und rechnerische Erklärungen für diese Form der kinetischen Racematspaltung findet man bei Eliel [67] und in der dort zitierten Literatur. Die Methode wurde z. B. zur Konfigurationbestimmung von Loganin [46], Lamiid [49] und Ajugosid [68] verwendet. Ihre Anwendbarkeit wird dadurch eingeschränkt, daß jeweils nur eine freie sekundäre Hydroxygruppe im Molekül vorhanden sein darf.

Die Konfiguration am C-1 kann nach dem van't Hoffschen „Prinzip der Superposition" $[67,69]$ bestimmt werden. Das C-Atom 1 eines Iridoids ist dem anomeren C-Atom einer Aldopyranose 
<smiles>CO[C@H]1O[C@H](CO)C(O)[C@H](O)[C@H]1O</smiles>

Methyl-B-D-

Glucopyranosid

$$
[M]_{D}=-66
$$

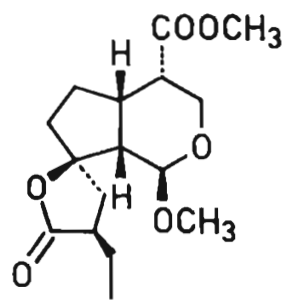

$[M]_{D}=-61$<smiles>CC(=O)OC1=CO[C@@H](O)[C@H]2[C@@H](O)[C@@H](O)C[C@]12O</smiles>

Abb. 17. Vergleidh von Molrotationen zur Bestimmung der Konfiguration an C-1 von Lamiid [49]<smiles>OC[C@H]1O[C@H](O)[C@@H](O)[C@H](O)[C@@H]1O</smiles>

B-D-Glucopyranose

$$
[M]_{D}=+34
$$<smiles>CO[C@H]1O[C@H](CO)C(O)[C@H](O)[C@@H]1O</smiles>

Methyl- $\alpha-D-$ Glucopyranosid

$[M]_{D}=+309$<smiles>CO[C@H]1OC[C@H](C(C)=O)[C@H]2CC[C@@]34OC(=O)C(I)[C@@H]3[C@]124</smiles>

$[M]_{D}=+431$<smiles>C[C@@H]1OC(CO)[C@@H](O)[C@H](O)[C@H]1O</smiles>

Methyl-B-DGlucopyranosid

$$
[M]_{D}=-66
$$<smiles>CC(=O)OC1C=CC(OC(C)=O)[C@]2(O)CC(O)[C@H](O)[C@H]12</smiles>

Lamiidaglykon

Lamiid

$[M]_{D}=+121^{\circ}$ 
weirgehend analog. Man hat daher die fü: die Konfigurationszuordnung von Zuckern gültigen Regeln auch auf die Iridoide übertragen: Bei allen untersuchten $\alpha$-D-Aldopyranosen zeigt das C-1 einen positiven Beitrag, bei allen untersuchten $\beta$-D-Aldopyranosen einen negativen Beitrag zur Molrotation des Moleküls. Man kann daher, wenn das Vorzeichen des Drehungsbeitrages für das C-1 bekannt ist, die Konfiguration am C- 1 daraus ableiten. Das Vorzeichen des Drehungsbeitrages erhält man bei Iridoiden entweder durch Vergleich zweier am C-1 epimerer Verbindungen, z. B. der bei der Hydrolyse von Iridoidglykosiden entstehenden Cyclohalbacetale bzw. der entsprechenden Methylacetale (Abb. 16), oder durch Vergleich eines Cyclohalbacetals bzw. Methylacetals mit dem entsprechenden Iridoidglykosid (Abb. 17). Die zuletzt genannte Zuordnung ist möglich, weil der Absolutwert der Molrotation mit zunehmender Größe des acetalisch gebundenen Restes zunimmt, während das Vorzeichen unverändert bleibt. In Analogie zu den D-Aldopyranosen schließt man dann aus einem negativen Beitrag des $\mathrm{C}-1$ zur Molrotation auf eine $\beta$-Stellung des OR-Restes und aus einem positiven Beitrag des $\mathrm{C}-1$ zur Molrotation auf eine $\alpha$-Stellung des ORRestes $[1,27,49]$.

\section{Literaturverzeichnis}

1. Büchi, G. u. R. H. Manning: Tetrahedron 18, 1049 (1962).

2. Fujise, S., H. Obara u. H. Uda: Chem. a. Ind. 289 (1960).

3. Birch, A. J., J. Grimshaw u. H. R. Juneja: J. Chem. Soc. 5194 (1961).
4. Wendt, M. W., W. Haegele, E. Simonitsch u. H. Schmid: Helv. Chim. Acta, 43, 1440 (1960).

5. Danielson, T. J., E. M. Hawes, C. A. Bliss: Can. J. Chem. 51, 1737 (1973).

6. Geissmann, T. A., W. F. Knaad, Jr. U. J. O. Knight: Tetrahedron Letters 1245 (1966).

7. Rimpler, H. u. H. Pistor: Z. Nacurforsch. $29 c, 368$ (1974).

8. Scarpati, M. L. u. M. Guiso: Terrahedron 23, 4709 (1967).

9. Takeda, Y., H. Nishimura u. H. Inouye: Phytochem. 14, 2647 (1975).

10. Tagushi, H. u. T. Endo: Chem. Pharm. Bull. (Tokyo) 22, 1935 (1974).

11. Esposito, P. u. M. L. Scarpati: Gazz. Chim. Ital. 100, 836 (1970).

12. Endo, T. u. H. Tagushi: Chem. Pharm. Bull. (Tokyo) 21, 2684 (1973).

13. Bobbitt, J. M., D. W. Spiggle, S. Mahboob, H. Schmid u. A. v. Philipsborn: J. Org. Chem. 31, 500 (1966).

14. Bobbitt, J. M., D. E. Kiely, A. Y. W. Lam u. E. I. Snyder: J. Org. Chem. 32, 1459 (1967).

15. Kitagawa, I., K. Hino, T. Nishimura, E. Iwata u. I. Yosioka: Chem. Pharm. Bull. (Tokyo) 19, 2534 (1971).

16. Asaka, Y., T. Kamikawa, T. Tokoroyama u. T. Kubota: Tetrahedron 26, 2365 (1970).

17. Panizzi, L., M. L. Scarpati u. G. Oriente: Gazz. Chim. Ital. 90, 1449 (1960).

18. Inouye, H., T. Yoshida, S. Tobita, K. Tanaka u. T. Nishioka: Tetrahedron Letters 2459 (1970).

19. Souzu, I. u. H. Mitsuhashi: 'Tetrahedron Lett. 191, (1970).

20. Inouye, H., T. Yoshida, Y. Nakamura u. S. Tobita: Tetrahedron Letters 4429 (1968).

21. Souzu, I. u. H. Mitsuhashi: Tetrahedron Letters 2725 (1969).

22. Inouye, H., T. Yoshida, S. Tobita, K. Tanaka u. T. Nishioka: Tetrahedron 30, 201 (1974).

23. Loew, P., C. v. Szczepanski, C. J. Coscia u. D. Arigoni: Chem. Commun. 1276 (1968).

24. Battersby, A. R., A. R. Burnett, G. D. Knowles u. P. G. Parsons: Chem. Commun.

25. Kamikawa, T., K. Inoue, T. Kubota u. M. C. Woods: Tetrahedron 26, 4561 (1970). 
26. Sévenet, T., C. Thal u. P. Potier: Tetrahedron 27, 663 (1971).

27. Halpern, O. u. H. Schmid: Helv. Chim. Acta 41, 1109 (1958).

28. Trim, A. R. u. R. Hill: Biochem. J. 50, 310 (1952).

29. Wieffering, J. H.: Phytochem. 5, 1053 (1966).

30. Bobbitt, J. M. u. K.-P. Segebarth in W. I. Taylor u. A. R. Battersby (Ed.): Cyclopentanoid Terpene Derivatives, New York 1969, Dekker.

31. Bianco, A., M. Guiso, C. Iavarone, R. Marini-Bettolo u. C. Trogolo: Gazz. Chim. Ital. 106, 733 (1976).

32. Karrer, P. u. H. Schmid: Helv. Chim. Acta 29, 525 (1946).

33. Bianco, A., M. Guiso, C. Iavarone, P. Passacantilli u. C. Trogolo: Gazz. Chim. Ital. 107, 83 (1977).

34. Sticher, O.: Helv. Chim. Acta 53, 2010 (1970).

35. Sticher, O.: Tetrahedron Lett. 291 (1975).

36. Guiso, M., A. Agostini u. R. Marini-Bettolo: Gazz. Chim. Ital. 104, 403 (1974).

37. Scarpati, M. L. u. P. Esposito: Gazz. Chim. Ital. 97, 1209 (1967).

38. Scarpati, M. L., M. Guiso u. P. Esposito: Gazz. Chim. Ital. 98, 177 (1968).

39. Rimpler H. u. B. Schäfer: Tetrahedron Lett. 1463 (1973).

40. Elphimoff-Felkin, I. u. P. Sarda: Tetrahedron Lett. 728 (1972).

41. Inouye, H., T. Arai u. M. Miyoshi: Chem. Pharm. Bull. (Tokyo) 12, 888 (1964).

42. Inouye, H., T. Yoshida, S. Tobita u. M. Okigawa: Tetrahedron 26, 3905 (1970).

43. Inouye, H. u. K. Fuji: Chem. Pharm. Bull. (Tokyo) 12, 901 (1964).

44. Inouye, H., T. Yoshida, Y. Nakamura u. S. Tobita: Chem. Pharm. Bull (Tokyo) 18, 1889 (1970).

45. Inouye, H., S. Saito u. T. Shingu: Tetrahedron Lett. 3581 (1970).

46. Battersby, A. R., E. S. Hall u. R. Southgate: J. Chem. Soc. (C) 721 (1969).

47. Lichti, H. u. A. von Wartburg: Helv. Chim. Acta 49, 1552 (1966).

48. Eigtved, P., S. R. Jensen u. B. J. Nielsen: Acta Chem. Scand. B. 28, 85 (1974).

49. Scarpati, M. L. u. M. Guiso: Gazz. Chim. Ital. 99, 1150 (1969).
50. Bentley, T. W., R. A. W. Johnstone u. J. Grimshaw: J. Chem. Soc. (C), 2234 (1967).

51. Weinges, K., P. Kloss u. W.-D. Henkels: Liebigs Ann. Chem. 759, 173 (1972).

52. Schilling, G., W.-D. Henkels, K. Künstler, K. Weinges, P. Kloss u. H. Jaggy: I.iebigs Ann. Chem. 230 (1975).

53. Lalonde, R. T., C. Wong u. A. I.-M. Tsai: J. Amer. Chem. Soc. 98, 3007 (1976).

54. Jolad, S., J. J. Hoffmann, R. M. Wiedhopf, J. R. Cole, R. B. Bates u. G. R. Kriek: Tetrahedron Lett. 4119 (1976).

55. Bock, K., S. R. Jensen u. B J. Nielsen: Acta Chem. Scand. B 30, 743 (1976).

56. Kubo, I., I. Miura u. K. Nakanishi: J. Amer. Chem. Soc. 98, 6704 (1976).

57. Lipnick, R. L.: Amer. Chem. Soc. 96, 2941 (1974).

58. Ouannes, C. u. J. Jacques: Bull. Soc. Chim. France 3601 (1965).

59. Eigtved, P., S. R. Jensen u. B. J. Nielsen: Acta Chem. Scand. B 28, 85 (1974).

60. Steyn, R. u. H. Z. Sable: Tetrahedron 27, 4429 (1971).

61. Bianco, A., M. Guiso, C. Iavarone u. C. Trogolo: Gazz. Chim. Ital. 105, 185 (1975).

62. Rimpler, H. u. J.-U. Junghanns: Z. Naturforsch. in Vorber.

63. a Danielson, T. J., E. M. Hawes u. C. A. Bliss: Can. J. Chem. 51, 760 (1973). b Bonadies, F., P. Esposito u. M. Guiso: Gazz. Chim. Ital. 104, 17 (1974).

64. Masaki, N., M. Hirabayashi, K. Fuji, K. Osaki u. H. Inouye: Tetrahedron Letters 2367 (1967).

65. Lentz, P. J. jun., u. M. G. Rossmann: Chem. Commun. 1269 (1969).

66. Horeau, A. u. H. Kagan: Tetrahedron 20, 2431 (1964).

67. Eliel, E. L.: Stereochemie der Kohlenstoffverbindungen, Weinheim 1966, Verlag Chemie.

68. Guiso, M., R. Marini-Bettolo u. A. Agostini: Gazz. Chim. Ital. 104, 25 (1974).

69. Fieser, L. F. u. M. Fieser: Organische Chemie, Weinheim 1965, Verlag Chemie.

Adresse: Prof. Dr. H. Rimpler, Institut für Pharmazentische Biologie, Schänzlestraße 1, D-7800 Freiburg Bundesrepublik Deutschland 